University of Nebraska - Lincoln

DigitalCommons@University of Nebraska - Lincoln

Stephen Ducharme Publications

Research Papers in Physics and Astronomy

$11-22-1997$

\title{
Low-field hole mobility in a photorefractive polymer
}

Arosha Goonesekera

University of Nebraska - Lincoln

Stephen Ducharme

University of Nebraska - Lincoln, sducharme1@unl.edu

James M. Takacs

University of Nebraska-Lincoln, jtakacs1@unl.edu

Lei Zhang

University of Nebraska - Lincoln

Follow this and additional works at: https://digitalcommons.unl.edu/physicsducharme

Part of the Physics Commons

Goonesekera, Arosha; Ducharme, Stephen; Takacs, James M.; and Zhang, Lei, "Low-field hole mobility in a photorefractive polymer" (1997). Stephen Ducharme Publications. 20.

https://digitalcommons.unl.edu/physicsducharme/20

This Article is brought to you for free and open access by the Research Papers in Physics and Astronomy at DigitalCommons@University of Nebraska - Lincoln. It has been accepted for inclusion in Stephen Ducharme Publications by an authorized administrator of DigitalCommons@University of Nebraska - Lincoln. 


\title{
Low-field hole mobility in a photorefractive polymer
}

\author{
Arosha Goonesekera and Stephen Ducharme \\ Department of Physics and Astronomy, Center for Materials Research and Analysis, University of \\ Nebraska, Lincoln, Nebraska 68588-0111 \\ James M. Takacs and Lei Zhang \\ Department of Chemistry, Center for Material Research and Analysis, University of Nebraska, Lincoln, \\ Nebraska 68588-0304
}

(Received 20 May 1997; accepted 17 September 1997)

We present the time-of-flight measurements of hole mobility in a photorefractive polymer composite as a function of temperature and applied electric field. The analysis shows that the temperature dependence of the low-field mobility is in apparent disagreement with the predictions of the Gaussian disorder model and also with polaron models. (C) 1997 American Institute of Physics. [S0021-9606(97)51744-2]

\section{INTRODUCTION}

The understanding of charge transport in molecularly doped polymers, xerographic photoreceptors in particular, has met with considerable progress in the past quarter-century. ${ }^{1-3}$ There has been remarkable success in the development of quantitative physical models of charge transport. The most notable and widely used models are the Gaussian disorder model (GDM) developed by Bässler and co-workers ${ }^{1}$ and the small polaron models ${ }^{4-6}$ though there are several other models worth consideration. ${ }^{6}$

The recent demonstrations of low-cost high performance photorefractive polymers ${ }^{7,8}$ has encouraged more detailed study of charge transport mechanisms because charge transport governs the response speed of these materials. Xerographic photoreceptors and photorefractive polymers are two different classes of molecularly doped polymers with similar components and similar charge transport physics. Due to these similarities, one expects there will be a good correlation between the transport mechanisms in these two systems. The photorefractive polymer generally consists of a host polymer, a nonlinear optical chromophore, a charge transport agent (acceptor or donor molecule) and a photosensitizing dye molecule, where each component has different function ${ }^{7}$ as described below.

The photorefractive effect is a reversible mechanism for formation of refractive index gratings (holograms) in electrooptic materials that has potential applications in integrated optics, optical data storage, optical computing and several other areas. ${ }^{9}$ Nonuniform illumination, for example, the interference fringes of intersecting coherent beams, generates free carriers in the bright regions and these carriers drift and diffuse into the darker regions where they are retrapped. This charge distribution in turn generates a space charge field which changes the index of refraction through the linear electro-optic response (the Pockels effect), creating a phase hologram with respect to the original intensity pattern. Unlike holograms recorded in photographic film, photorefractive holograms need no development and can be erased and/or recorded over. The speed of the photorefractive effect is proportional to the photoconductivity; research emphasis on charge transport mechanisms is therefore a vital component in the study of photorefractive grating dynamics and for improving device performance.

Recently, we have conducted an investigation of hole mobilities using, standard time-of-flight (TOF) techniques, in the photorefractive polymer bisphenol $A$ 4-4'-nitroaminostilbene (bisA-NAS) doped with the hole transport agent diethylamino-benzaldehyde-diphenyl hydrazone (DEH). ${ }^{10}$ Several unique features revealed by this investigation ${ }^{10}$ have led us to examine the data in greater detail in light of the Gaussian disorder model. In this new analysis we paid particular attention to the low-field mobility, which exhibits a temperature dependence in apparent disagreement with the predictions of the Gaussian disorder model and also with small polaron models.

\section{EXPERIMENT}

The samples for the mobility measurements, were prepared as follows: First, a $45 \mathrm{~nm}$ thick $\alpha$-Se layer was vapor deposited on an indium tin oxide (ITO) coated glass substrate. Then a polymer solution containing bisA-NAS polymer and hole transport agent DEH $(\sim 30 \%$ by weight $)$ in 1-methoxy-2-propanol was coated by solvent spinning on top of the $\alpha$-Se layer. The thickness of the polymer layer was $\sim 400 \mathrm{~nm}$, determined from ellipsometry and capacitance measurements. The polymer-coated glass plates were placed on a hot plate at $40{ }^{\circ} \mathrm{C}$, in argon for $15 \mathrm{~h}$ to remove the remaining solvent. Finally, after spin coating a polyvinyl alcohol (PVA) buffer layer of $\sim 300 \mathrm{~nm}$ thickness on the polymer, a $40 \mathrm{~nm}$ thick $\mathrm{Ni}$ electrode was vapor deposited in vacuum on top. Further details of the sample preparation and polymer synthesis are found in Ref. 11.

The mobility measurements were made by the conventional time-of-flight technique, where a sheet of holes was injected to the doped polymer layer through photoexcitation in the $\alpha$-Se layer by exposure to $532 \mathrm{~nm}$ second-harmonic radiation from a pulsed Nd-YAG laser with a pulse duration of 4-6 ns. The sample was mounted on a copper block maintained at constant temperature stable to $\pm 0.1 \mathrm{~K}$. The photocurrent transients with positive bias voltage (Fig. 1) were 

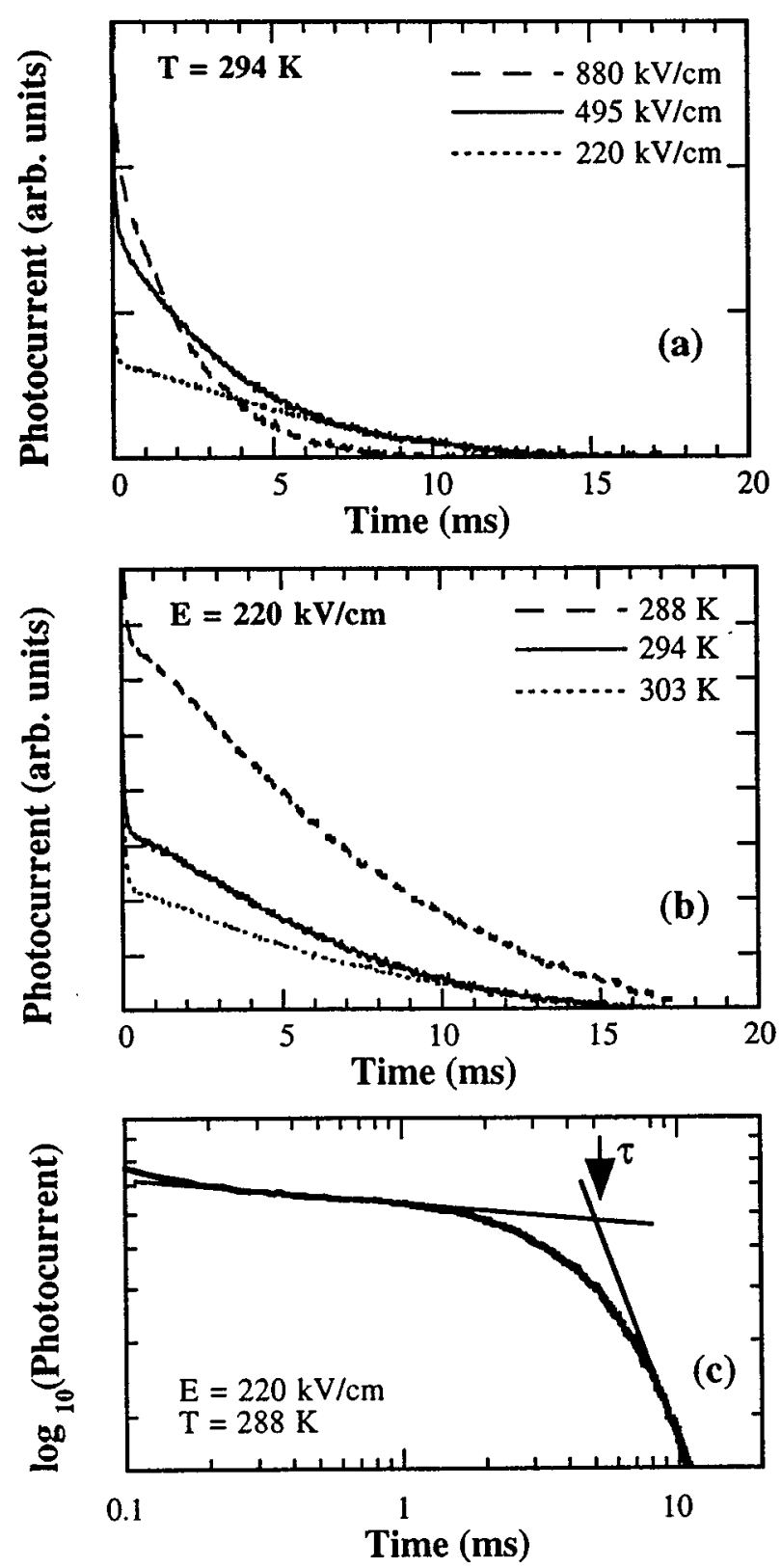

FIG. 1. The time-of-flight photocurrent transients (a) at different fields and at temperature $294 \mathrm{~K}$ and, (b) at different temperatures and at field 220 $\mathrm{kV} / \mathrm{cm}$. The $\log -\log$ plot (c) for field $220 \mathrm{kV} / \mathrm{cm}$ and temperature $288 \mathrm{~K}$ allows determination of the transit time in the presence of strong dispersion.

recorded with a digital oscilloscope connected to a resistor in series with the sample and the voltage source. There was no observable electron current when the experiment was conducted with negative bias voltage. A negligible amount of charge was depleted from the positive electrode and therefore the sample voltage was constant during the measurements. The transit times were determined from the intersection of the asymptotes of the curve obtained from the log$\log$ plot of the photocurrent transients [Fig. 1(c)]. Further details on the time-of-flight method and transient time determination are found in Refs. 6 and 10.

The mobility $\mu$ of the charge carriers is obtained from the familiar equation for carrier mobility at constant electric field $E=V / L, \mu=($ average drift speed $/ E)=L^{2} / \tau V$, where $L$ is the thickness of the polymer layer, $\tau$ is the transit time of the charge carriers, and $V$ is the dc voltage across the polymer layer. The dielectric constant of the polymer mixture is $2.7 \pm 0.3$ and dielectric constant of PVA is $2.1 \pm 0.2$ at $1 \mathrm{kHz}$. The sample capacitance was $2.1 \mathrm{nF}$ at $1 \mathrm{kHz} .^{10}$ The above data was used to determine the voltage across the polymer layer.

A notable experimental observation is that the photocurrent transients (Fig. 1), show a transition from weakly dispersive to highly dispersive shapes as the applied field is increased, but the shape of the photocurrent transients does not seem to depend on temperature. The transient plots do not appear to have either the Scher-Montroll form or the pre-transit time dispersion form, which would indicate Gaussian velocity dispersion, as recently reported for an organic semiconductor. ${ }^{12}$

\section{RESULTS AND DISCUSSION}

The mobility depends strongly on electric field, a characteristic feature in molecular materials, as shown in Fig. 2. The most striking feature is the minimum in the mobility at a moderately high electric field $E_{\min } \sim 600 \mathrm{kV} / \mathrm{cm}$. The position of this minimum $E_{\min }(T)$ decreases with increasing temperature, as shown in the inset of Fig. 2. At high fields above $E_{\min }$ our data reasonably fit an $\exp \left(\beta E^{1 / 2}\right)$ "PooleFrenkel" dependence, with a temperature dependence consistent with the Gaussian disorder model (GDM). At high fields, according to the GDM, the electric field and temperature dependence of the mobility is given (only above $E_{\min }$ ) by

$$
\mu(T, E)=\mu_{0} \exp \left[C\left\{\left(\frac{w}{k_{B} T}\right)^{2}-\Sigma^{2}\right\} E^{1 / 2}-\left(\frac{2 w}{3 k_{B} T}\right)^{2}\right],
$$

where $w$ is the width of the hopping site Gaussian energy distribution and $\Sigma$ similarly measures the Gaussian positional disorder. $\mu_{0}$ is the prefactor mobility and $k_{B}$ is the Boltzmann constant. Equation (1) is valid only at high fields $E$ $>E_{\min }$ where energetic disorder dominates. ${ }^{2}$ The decrease of $E_{\min }$ with increasing temperature follows from the temperature dependence of Eq. (1) and the weak low-field temperature dependence of the mobilities. The Gaussian energy width $w=0.095 \pm 0.005 \mathrm{eV}$ and Gaussian positional disorder parameter $\Sigma=2.67 \pm 0.23$ have been determined by extrapolating the high field data. ${ }^{10}$ It is important to note here that the curves shown in Fig. 2 are similar to the results of Monte Carlo simulations conducted by Bässler et al. ${ }^{2}$ for the fitting parameters $w / k_{B} T \sim \Sigma \sim 3$ also in good agreement with the model. The empirical constant $C=3.13 \pm 0.11$ $\times 10^{-4}(\mathrm{~cm} / \mathrm{V})^{1 / 2}$ is within the range $2.9-3.3 \times 10^{-4}$ $(\mathrm{cm} / \mathrm{V})^{1 / 2}$ found in a wide range of molecularly doped polymers. ${ }^{6}$

At low fields (below $E_{\min }$ ) the functional form of the field dependence has not been forthcoming from the existing models. In accordance with the disorder theory, the decrease in mobility with increasing fields up to the turning point 


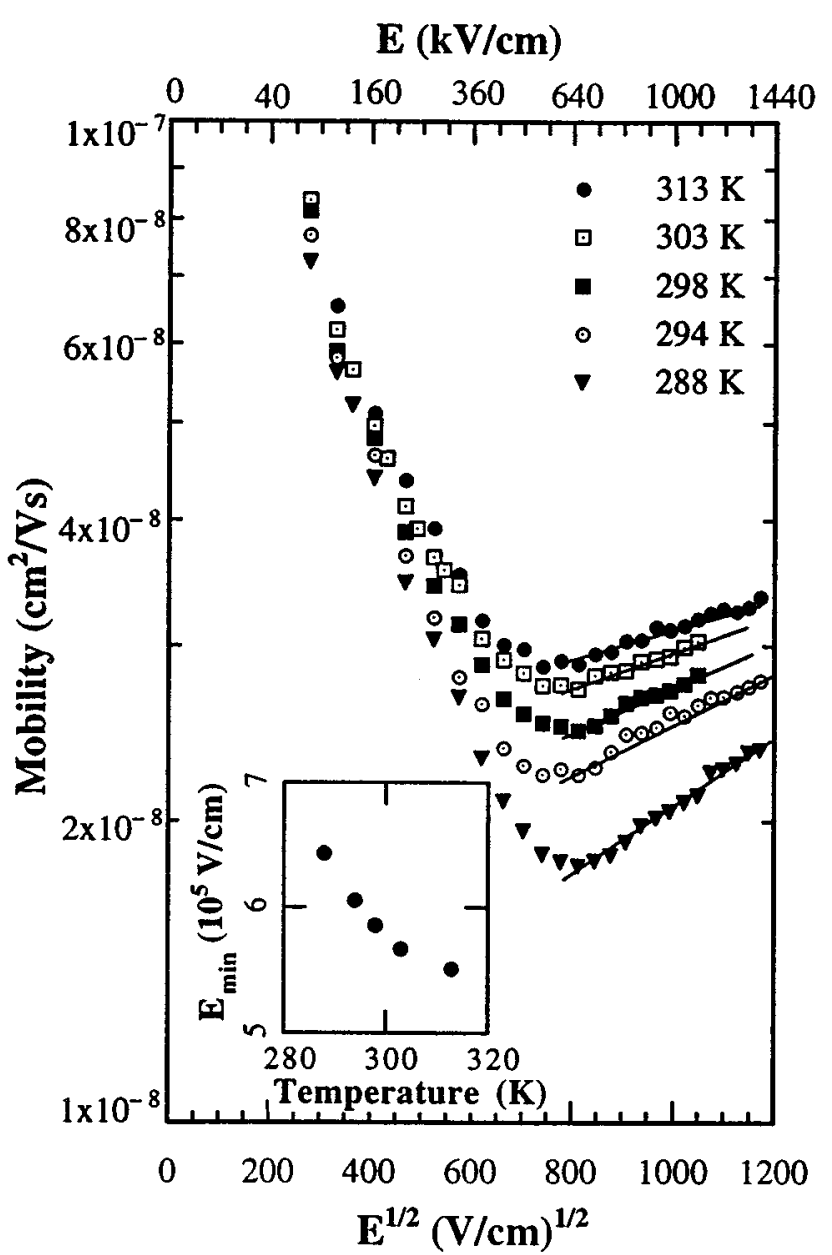

FIG. 2. The logarithm of mobility showing a minimum in the mobility $E_{\min }$ and the Poole-Frenkel dependence at high fields. The solid lines are fits to Eq. (1). The inset shows the variation of the $E_{\min }$ with temperature.

$\left(E_{\min }\right)$ can be attributed to the dominance of positional disorder and the increase in mobility with increasing field above $E_{\min }$ to the dominance of energetic disorder. ${ }^{2}$ Even though this negative field dependence (below $E_{\text {min }}$ ) has been observed in both experiment ${ }^{2,12-14}$ and Monte Carlo simulations of the GDM, ${ }^{2}$ these reports did not explain the temperature dependence at low fields. The data in Fig. 2 covers a wider range of fields below $E_{\text {min }}$ than in the previous reports and clearly shows a trend of decreasing negative slope with increasing temperature.

Figure 3 shows that the activation energy below $E_{\text {min }}$ appears to vanish near zero field, or more precisely, the temperature dependence of the log of the mobility vanishes, consistent with pure diffusion-dominant charge transport without activation. In these plots $\Delta_{1}$, the slope of the $\ln (\mu)$ vs $T^{-1}$ plots represents the traditional activation energy and $\Delta_{2}$, the square root of the slope of $\ln (\mu)$ vs $T^{-2}$ plots represents an alternate definition germane to the GDM. Also in the low-field regime, the slope $\beta$ of $\ln (\mu)$ vs $E^{1 / 2}$ plots is negative and its magnitude decreases with increasing temperatures. Several recent studies of molecularly doped polymers showing the low-field decrease in mobility, all show nonzero activation as the applied field tends to zero, ${ }^{15,16}$ in

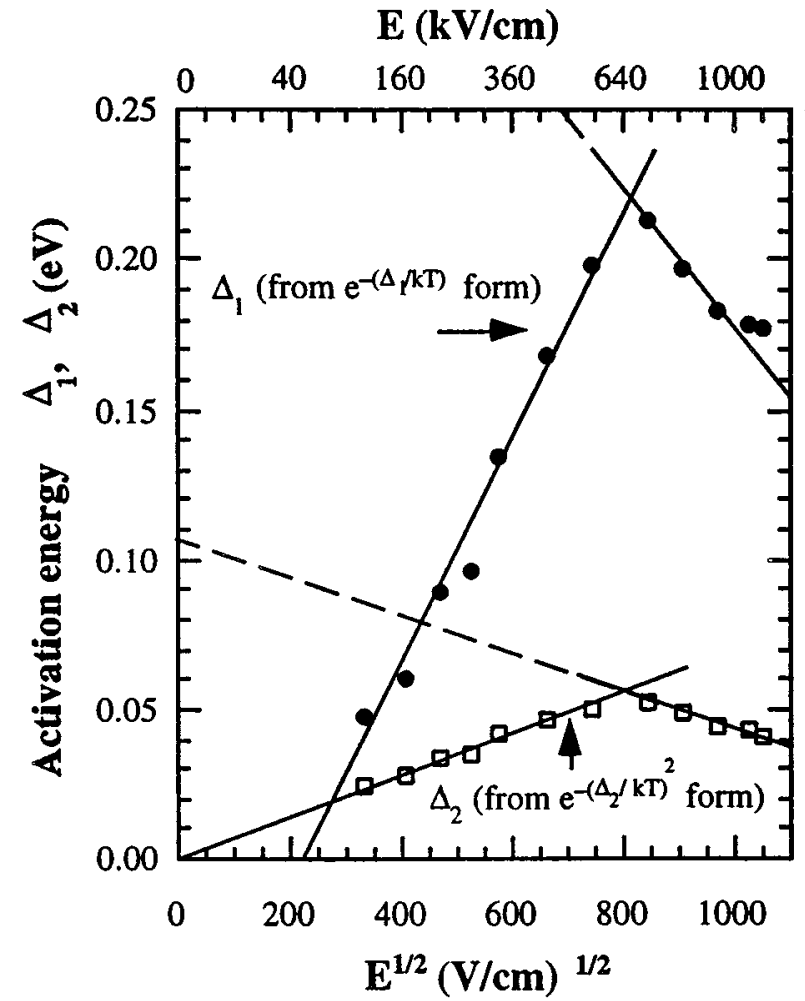

FIG. 3. The activation energy vs $E^{1 / 2}$. The activation energy $\Delta_{1}$ is derived assuming the conventional from $\exp \left(-\Delta_{1} / k T\right)$ form and activation energy $\Delta_{2}$ is derived from assuming the $\exp \left[-\left(\Delta_{2} / k T\right)^{2}\right]$ form appropriate to the Gaussian disorder model.

contrast to our present results. Note that we have measured the mobility for a very limited range of temperatures (279$318 \mathrm{~K}$ ) due to the low $T_{g} \sim 308-313 \mathrm{~K}$ of the polymer system and therefore could not reliably determine if there is a prefactor of the form $T^{-1}$ expected in a pure diffusion regime or $T^{p}$ expected from polaron models. ${ }^{6}$ The apparent activation energies $\left(\Delta_{1}\right.$ or $\left.\Delta_{2}\right)$ are readily determined over a limited temperature range because the mobility depends on them exponentially.

The question of low-field thermal activation of the carrier mobility in the GDM is an important one. As has already been pointed out, the negative slope of the low-field mobility is attributed to positional disorder through the elimination of the transport channels with "uphill" (against the field) hops. However, the remaining "downhill" paths will still exhibit activation even at zero field, through their Miller-Abrahams hopping probabilities. ${ }^{17}$ The data in Fig. 3 show clearly that the apparent activation energy tends to vanish at nonzero field (for the Arrhenius $\exp \left[-\left(\Delta_{1} / T\right)\right]$ form) or at zero-field (for the $\exp \left[-\left(\Delta_{2} / k T\right)^{2}\right]$ form appropriate to the GDM).

Another striking feature of the GDM activation energy $\Delta_{2}$ is that it rises with $E^{1 / 2}$ from zero field followed by a decrease for fields above $E_{\min }$ where Eq. (1) is expected to hold. The slope of $\Delta_{2}$ has approximately the same magnitude, but opposite sign above and below $E_{\min }$. At this time we have no physical interpretation suggesting this is more than a coincidence, but the question deserves further study. 


\section{CONCLUSIONS}

The apparent vanishing of the temperature dependence of the mobility at zero field suggests that the activation energy (Fig. 3) also vanishes, in contradiction to the MillerAbrahams hopping probability. ${ }^{17}$ Also, the slope $\beta$ of lowfield $\ln (\mu)$ vs $E^{1 / 2}$ plots increases with decreasing temperature is not explainable by the results of recent Monte Carlo simulations of the Gaussian disorder model ${ }^{2}$ and in contradiction with measurements in several other molecularly doped polymers. ${ }^{2,12,13}$ The polaron models also fail to give a reasonable explanation for the results of low-field mobility as they require thermal activation at zero field. Therefore, these results pose a challenge to both Miller-Abrahams based and polaron models.

\section{ACKNOWLEDGMENTS}

This work was supported by the National Science Foundation, by NSF/Nebraska EPSCOR, by the Air Force office of Scientific Research, and by the Nebraska Research Initiative through the Center for Materials Research and Analysis. We thank Paul Borsenberger, David Dunlap, and Alexander Bune for useful advice.
${ }^{1}$ H. Bässler, Phys. Status Solidi B 175, 15 (1993).

${ }^{2}$ P. M. Borsenberger, L. Pautmeier, and H. Bässler, J. Chem. Phys. 94, 5447 (1990)

${ }^{3}$ L. B. Schein, Philos. Mag. B 65, 795 (1992)

${ }^{4}$ D. Emin, Phys. Rev. Lett. 24, 303 (1974).

${ }^{5}$ L. B. Schein, D. Glatz, and J. C. Scott, Phys. Rev. Lett. 65, 472 (1990).

${ }^{6}$ P. M. Borsenberger and D. S. Weiss, Organic photoreceptors for imaging systems (Marcel Dekker, New York, 1993).

${ }^{7}$ S. Ducharme, R. W. Twieg, J. C. Scott, and W. E. Moerner, Phys. Rev. Lett. 66, 1846 (1991).

${ }^{8}$ K. Meerholz, B. L. Volodin, Sandalphon, B. Kippelen, and N. Peyghambarian, Nature 371, 497 (1994).

${ }^{9}$ Photorefractive Materials and Their Applications I: Fundamental Phenomena, Vol. 61, edited by P. Günter and J.-P. Huignard (Springer Verlag, Berlin, 1988).

${ }^{10}$ A. Goonesekera, S. Ducharme, J. M. Takacs, and L. Zhang, in Xerographic Photoreceptors and Photorefractive Polymers, Vol. 2850, edited by S. Ducharme and J. W. Stasiak (SPIE, Bellingham, WA, 1996), pp. 41-52.

${ }^{11}$ B. E. Jones, S. Ducharme, M. Liphardt, A. Goonesekera, J. M. Takacs, L. Zhang, and R. V. Athalye, J. Opt. Soc. Am. B 11, 1064 (1994).

${ }^{12}$ A. Ioannidis, M. F. Lawrence, H. Kassi, R. Cote, J. P. Dodelet, and R. M. Leblanc, Chem. Phys. Lett. 205, 46 (1993).

${ }^{13}$ P. M. Borsenberger and H. Bässler, Phys. Status Solidi B 170, 291 (1992).

${ }^{14}$ S. Heun and P. M. Borsenberger, Physica B 216, 43 (1995).

${ }^{15}$ T. Sasakawa, T. Ikeda, and S. Tazuke, J. Appl. Phys. 65, 2750 (1989).

${ }^{16}$ M. A. Abkowitz, Philos. Mag. B 65, 817 (1992).

${ }^{17}$ A. Miller and E. Abrahams, Phys. Rev. 120, 745 (1960). 